Itinéraires Itinéraires

Littérature, textes, cultures

2014-1 | 2015

Textualités numériques

\title{
Fragmentation et saisissement pornographiques
}

Une approche par les légendes des vignettes des sites web

\section{François Perea}

\section{CpenEdition}

Journals

Édition électronique

URL : http://journals.openedition.org/itineraires/2335

DOI : $10.4000 /$ itineraires.2335

ISSN : 2427-920X

Éditeur

Pléiade

Référence électronique

François Perea, «Fragmentation et saisissement pornographiques », Itinéraires [En ligne],

2014-1 | 2015, mis en ligne le 15 janvier 2015, consulté le 01 mai 2019. URL : http://

journals.openedition.org/itineraires/2335; DOI : 10.4000/itineraires.2335

Ce document a été généré automatiquement le 1 mai 2019.

\section{(c)}

Itinéraires est mis à disposition selon les termes de la licence Creative Commons Attribution - Pas

d'Utilisation Commerciale - Pas de Modification 4.0 International. 


\title{
Fragmentation et saisissement pornographiques
}

\author{
Une approche par les légendes des vignettes des sites web
}

\section{François Perea}

La chair y est à la fois exhibée et absente.

(Jean-Claude Guillebaud 2011:151)

1 Sur le net, les sites au contenu pornographique présentent une grande diversité, depuis les sites à accès payant (tels ceux des producteurs de films par exemple) jusqu'aux sites « de niche » présentant des contenus ultra-spécialisés, en passant par les blogs érotiques d'internautes exhibitionnistes.

Le corpus sur lequel repose notre étude est extrait de sites pornographiques particuliers : gratuits et populaires (ils génèrent le plus de trafic et apparaissent en tête dans les résultats des moteurs de recherche), ils proposent des extraits vidéographiques d'une durée variant de quelques secondes à plusieurs dizaines de minutes. Ces derniers sont postés pour une majorité écrasante par des producteurs de films jadis classés X (qui en font ainsi la promotion et proposent des liens vers des versions plus longues et payantes). Ils sont parfois proposés par les internautes eux-mêmes, conformément à l'esprit du Web 2.0, ses stratégies d'exposition de soi et les injonctions à ce que Tisseron ${ }^{1}$ a pu appeler « extime »: «Je propose d'appeler "extimité" le mouvement qui pousse chacun à mettre en avant une partie de sa vie intime, autant physique que psychique » (2001:52).

L'ensemble participe d'un mouvement global de mise On/scène de l'obscène (Williams 2004), c'est-à-dire, d'une exposition à la vue publique de scènes traditionnellement réservées à la sphère intime. La confusion entre productions professionnelles et personnelles est d'ailleurs stratégiquement entretenue: les sites observés mettent en avant les insignes du Web social et participatif (notes et avis des internautes, etc.) et les productions industrielles que l'on y trouve majoritairement n'hésitent pas à mettre en scène des acteurs (pseudo-amateurs) et des techniques de réalisation (fausses webcams par exemple) singeant le partage et l'échange de films personnels. Ainsi, notamment, les références commerciales disparaissent au profit d'un 
réseautage sans tabou mis en scène (des publicités apparaissent sous forme de pop-ups qui imitent les échanges sur les chats).

4 C'est dans ce contexte que nous nous attachons à l'étude des légendes accompagnant les vignettes dans ces sites pornographiques également appelés tubes. Une première partie s'attachera à en décrire la nature et les fonctionnements (catégorisation par pornotypes, agglutinement en pseudo-récits). Une seconde partie observera leur participation aux scripts de la sexualité (Gagnon et Simon) et le travail de la fragmentation de ces discours. Pour conclure, nous nous interrogerons sur la place de ces légendes et de leurs caractéristiques dans le cadre de l'interpellation émotionnelle visée par l'interpellation pornographique.

\section{Légendes, pornotypes}

\subsection{Des légendes}

5 Au premier regard, la page d'accueil se présente comme un mur de vignettes (thumbnails) qui sont autant de liens vers les extraits vidéographiques. La plupart de ces vignettes ne se distinguent guère les unes des autres: les motifs, les couleurs, les cadrages sont souvent les mêmes et elles composent, placées côte à côte dans la grille, un patchwork quasi homogène dont la page d'accueil est le centon.

6 Chaque vignette est accompagnée d'une courte légende visant à préciser le contenu de l'extrait. Celle-ci est prise dans un double jeu de référence :

(a) référence, d'abord, à la vignette qui est une des images de l'extrait vidéographique ;

(b) référence, ensuite (et peut-être surtout) aux éléments qui figurent dans le menu " catégories » (ou categories, ou category dans les sites anglophones).

7 Cette seconde référence (b) a toute son importance : le lexique est en effet souvent propre au milieu et l'internaute pornophile ne saura interpréter les légendes qu'en fonction de son expérience de navigation qui lui permettra de saisir les acronymes («MILF », de Mother I'd like to fuck, désigne une femme mariée mère de famille), sigles («BBW », de Big Big Woman) et autres lexèmes renvoyant à des pratiques spécialisées («bukkake »). Ainsi, ce n'est parfois qu'en fonction de ces catégories que des légendes telles que «MILF BBW get bukkaked » pourront être entièrement comprises².

8 La référence de la légende à la vignette (a) est bien entendu primordiale pour l'internaute: elle lui permet de mieux cibler ses choix. Ces légendes précèdent, historiquement, les images. Pendant longtemps, alors que les sites pornographiques ne proposaient pas de vignettes pour éviter des temps de chargement des pages trop longs, les ancêtres des pages que nous observons ici se contentaient de listes descriptives composées de ces légendes. L'introduction de l'image fixe, puis parfois mobile (les vignettes s'animent sur certains sites lors de leur survol par le pointeur) a donc modifié le statut de ces textes: autonomes à l'origine, ils sont devenus des légendes accolées aux images. Cette évolution a des conséquences syntaxiques (passage de phrases verbales à des phrases nominales, peut-être sur le modèle des nuages des tags, nous y reviendrons) qui interrogent le statut et la nature du message linguistique dans la production multimédiatique.

Dans un contexte où l'image et l'attraction scopique paraissent être les ressorts essentiels et annihilateurs des autres codes (l'internaute visite le site pour voir), nous remarquons 
alors l'importance de ces messages linguistiques. Importance historique d'abord, puisqu'ils ont précédé le lien par l'image et ont façonné les usages de navigation, cartographié et légendé le territoire de la monstration pornographique. Importance signifiante ensuite, puisqu'ils portent la promesse de ce qu'il y a à voir après le clic. Importance modélisatrice, enfin, puisqu'ils sont les révélateurs d'un imaginaire travaillé et pris dans les rets de la codification pornographique. menus " catégories ", auxquels nous avons consacré une précédente étude (Perea 2012), menée sur un corpus de 761 occurrences dans les menus de 13 sites pornographiques ( 9 anglophones et 5 francophones, ces derniers étant souvent des calques des premiers) : youporn.com, pornhub.com, video-2-cul.com, goziga.com, mustvideos.com, pornstream.fr, qualisexe.com, porn365.com, 89.com, orgasm.com, freeporn.sex, xxdessert.com, xxxvogue.net, tube8.com, pornvanity.com. Un pointage réalisé en octobre 2011, un an après la constitution $\mathrm{du}$ premier corpus, auprès des cinq sites arrivant (à cette nouvelle période) en tête du classement du moteur de recherche Google montre la stabilité de ces menus et de la distribution des classes de base. Soulignons que ces sites sont "généralistes", à orientation hétérosexuelle, une prochaine étude devant prendre pour objet les sites (auto-)déclarés gays.

La surabondance de catégories - jusqu'à 117 pour un seul site, 58 en moyenne dans notre corpus - est particulièrement remarquable. Les autres supports des productions pornographiques (magazines, films) n'avaient jamais procédé à une telle atomisation quasi-fétichiste des pratiques, se contentant de préciser les genres concernés (dans les productions alors qualifiées de «hétéro», "gays", «lesbienne», «bi» ou «transsexuelle ») et quelques «niches» spécialisées («SM » par exemple). Au contraire, ces sites généralistes réalisent la conjonction entre les courants «mainstream or margin » (Williams 2004), les niches commerciales. 
Le foisonnement des catégories est peut-être lié à la navigation anonyme possible sur le net : le spectateur, libéré du regard du vendeur et des autres clients, pouvant explorer les formes les plus imprévues de la sexualité sans risque. Il permet, de plus, de proposer un nombre presque infini de nouveaux créneaux commerciaux et de les promouvoir sans peine grâce à la chaîne des « tags » qui permet de les retrouver d'un site à l'autre.

Les menus renvoient ainsi (i) aux caractéristiques exposées des personnages («black», «big tits », " young ", « amputee ») (ii) à leurs relations et activités sexuelles (« anal», «blowjob", «BDSM », " voyeur ») ou, plus rarement (iii) aux procédés technologiques et genres du site (" webcam », « mangas », " gonzo »).

Fig. 1. Classification des groupes de pornotypes

\begin{tabular}{|c|c|c|c|}
\hline \multicolumn{2}{|c|}{ (1) Pornotypes féminins } & \multicolumn{2}{|c|}{$\begin{array}{l}\text { (2) Pornotypes liés aux actes organiques (et } \\
\text { substituts), sécrétions }\end{array}$} \\
\hline $\begin{array}{l}\text { Organes } \\
\text { ex.: Big Butt, } \\
\text { Deep Throat, } \\
\text { Pussy, Ass, etc. }\end{array}$ & $\begin{array}{l}\text { Caractéristique physique } \\
\text { / psychologique } \\
\text { ex.: Young, Uniform, } \\
\text { Shemale, Fat, Asian, } \\
\text { Amputée, Horny, etc. }\end{array}$ & $\begin{array}{l}\text { Actes } \\
\text { ex. : Blow Job, Anal, } \\
\text { Double penetration, } \\
\text { Spanking, Dildo, etc. }\end{array}$ & $\begin{array}{l}\text { Productions } \\
\text { Cumshot, Lactating, } \\
\text { Pissing, Facial, Enema, } \\
\text { Bukkake, etc. }\end{array}$ \\
\hline (3) Pornotypes m & asculins & $\begin{array}{l}\text { (4) Pornotypes liés } \\
\text { relationnelle }\end{array}$ & à l'orientation \\
\hline $\begin{array}{l}\text { Apparence } \\
\text { physique } \\
\text { (rares) } \\
\text { ex. : Old, Black }\end{array}$ & $\begin{array}{l}\text { Organes } \\
\text { ex. : Big Dick }\end{array}$ & \begin{tabular}{|lr} 
Préférences & \\
ex. : & BDSM, \\
Exhibitionism, Gang \\
Band, Glory & Hole, \\
Lesbian, etc. &
\end{tabular} & $\begin{array}{l}\text { Contextes } \\
\text { ex.: Outdoor, Drunk, } \\
\text { Sex at work, } \\
\text { Secretary, } \\
\text { etc. }\end{array}$ \\
\hline
\end{tabular}

Ces regroupements peuvent bien entendu être discutés : les pôles présentés ne constituent pas des groupes étanches les uns aux autres.

17 Ces classements opèrent une déconstruction des éléments représentés et offrent un mode de représentation particulier. Au-delà des stéréotypes, l'entreprise pornographique de monstration repose ainsi sur ce que nous avons appelé des pornotypes, qui consistent en une atomisation catégorielle qui, plutôt que proposer une image globale et simplifiée du personnage ou de l'action, le réduit à un trait prégnant, rendu saillant et représentatif par une sorte de réduction métonymique (par exemple: Fat/BBW, Drunk ou Redhead). Soulignons, pour lever l'ambiguïté terminologique, que Paveau (2011) utilise le terme pornème(s) pour désigner certains sigles et acronymes dans le discours pornographique.

18 Avec le pornotype, chacun peut trouver un trait captant son intérêt ou sa curiosité parfois incrédule, dans ces ensembles codés où se côtoient tous les traits imaginables ( " bourrée », « dwarf ») et variés ( " young », « MILF », « granny »).

19 Les pornotypes sont donc des opérations discursives de catégorisation qui reposent sur un imaginaire et des représentations propres au X. Ils participent, au-delà ou en même temps que la constitution de niches commerciales, à l'élaboration d'objets médiaticocognitifs spécifiques, au-delà des stéréotypes que l'industrie de la pornographie doit dépasser car ils ne suffisent pas à différencier ses produits des œuvres érotiques ou 
publicitaires. Ces objets offrent alors au sujet des points d'achoppement à son désir, selon Patterson: «En réalité, ce que tend à offrir la cyberpornographie [...] est un environnement dans lequel le désir et la position du sujet sont produits comme des "vérités" de soi à travers un discours de catégorisation et de classification [...]» (2004 : 106 ; nous traduisons).

\subsection{Syntaxe des légendes}

Les légendes apparaissent comme des combinaisons de signes au rang desquels les pornotypes figurent en bonne place. Pour observer ces combinaisons, nous nous appuyons ici sur un corpus de 98 légendes extraites des pages d'accueil de cinq sites pornographiques (deux francophones/français et trois anglophones/américains).

Il est intéressant, pour commencer, de souligner les disparités dans notre corpus. On remarque en effet de grandes différences entre les sites français et les sites américains : les premiers privilégient des légendes articulées à un verbe (plus particulièrement à la voix passive) alors que les seconds affichent des légendes nominales.

Fig. 2. Répartition des légendes (pourcentages)

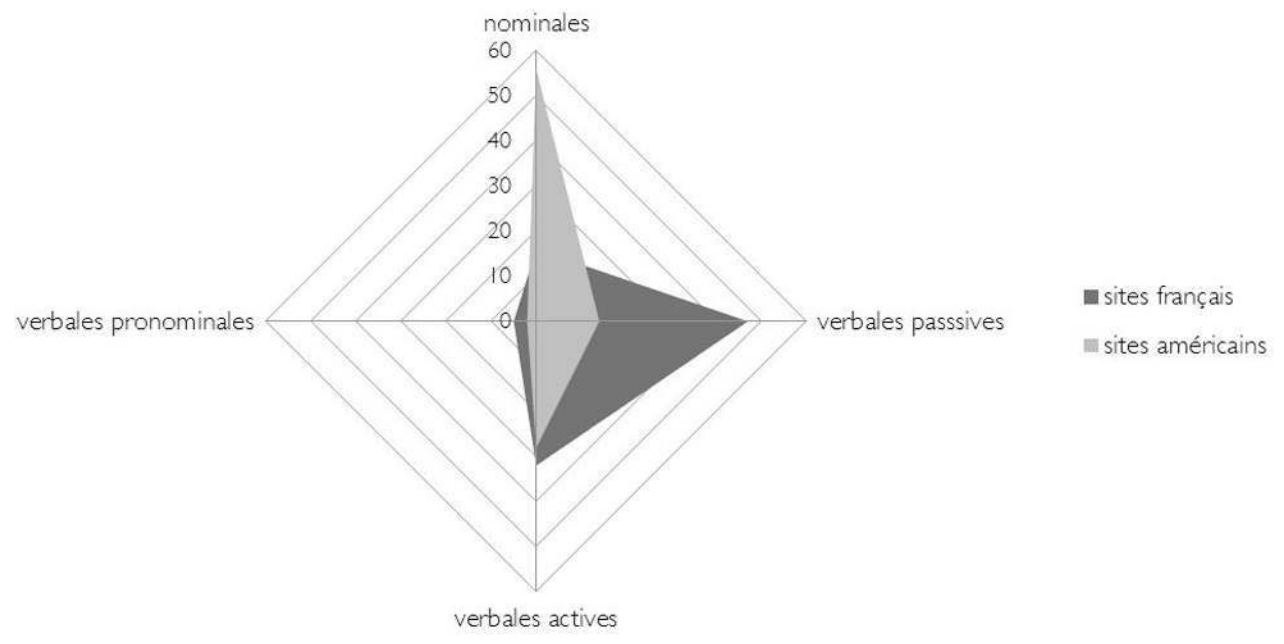


donc pas surprenant de voir la part des phrases nominales augmenter au fil des années sur les sites pornographiques français.

Les légendes nominales reposent sur une succession de pornotypes: "Horny MILF Anal Interracial», "Granny handjob and tit fuck», «Baise lesbienne avec des blondes plantureuses", "Partouze géante de matures et beurettes torrides aux seins volumineux », etc.

Le principe repose sur une double stratégie d'interpellation.

Interpellation du sujet pornophile, d'abord, par un saisissement de ses déterminations intimes via les mots-clés des pornotypes. Ainsi, les personnes traitées pour des addictions à la pornographie soulignent la recherche de visionnage de pratiques de plus en plus " extrêmes » (Kavciyan, Rossé et Codina 2008) pour lesquelles les pornotypes servent de points de repères. Cela est d'autant plus vrai que les sujets sont soumis à une excitation sexuelle : Ariely et Loewenstein (2006) en ont exploré un aspect en demandant à des étudiants de Berkeley de répondre à des questionnaires sur un ordinateur, grâce à un dispositif « using only the non-dominant hand » et en se masturbant. Le groupe d'étudiants auto-excités était plus enclin à avoir des relations sexuelles avec des filles de 12 ans, des femmes de 60 ans, des hommes, des personnes extrêmement grosses, etc., qui correspondent à des pornotypes (" young ", « granny ", " gay ", « BBW », etc.).

L'utilisation des noms permet également de répondre à l'impératif de référencement des extraits : les liens ainsi attachés à des mots-clés, répétés d'un site à l'autre, entrent dans la chaîne quantitative de la reconnaissance par les algorithmes de recherche: l'interpellation du robot de référencement est ainsi un «bénéfice secondaire » de ces légendes nominales.

Les phrases verbales à la voix passive (ou tournures équivalentes) sont majoritaires sur les sites français (elles représentent près de la moitié des légendes). Leur forme est extrêmement stéréotypée : patiente - verbe - (patiente) - (agent M) - (circonstance).

Ainsi : « Pucelle - se fait défoncer - (le cul) - (à coup de béquille et de bite) - (dans une cave) »(nous rajoutons parenthèses et tirets pour révéler le format).

Le personnage féminin est l'élément essentiel dans ces légendes: placé en début de phrase, souvent largement défini (par exemple : « Une bombasse brune aux gros nibars se fait culbuter »), il est, d'un point de vue syntaxique, en position de patient qui subit l'action. Les autres éléments étant optionnels, il apparaît que la mise en avant (1) du personnage féminin et (2) de l'acte subit, soit le dénominateur commun de ces légendes (verbales ou nominales). Notons que la soumission féminine est largement accentuée par le choix des verbes : défoncer, éclater, exploser, etc.

2 Les phrases verbales à la voix active représentent un tiers des légendes des sites français et américains de notre corpus. Le personnage féminin (qui devient actif) reste antéposé au verbe et ipso facto, demeure l'élément premier. Le personnage masculin est quant à lui drastiquement réduit, le plus souvent à son sexe. Ainsi : «BBW handles two hard dicks in her hole».

33 Les phrases à la voix pronominale sont rares et renvoient le plus souvent à des scènes ne mettant en scène que des femmes.

Quelles que soient leurs tournures syntaxiques (nominale ou verbale), les légendes mettent en avant des éléments obligatoires et des éléments optionnels. 


\subsection{Pseudo-récit} situation.

Nous ne le pensons pas. $(2003: 27)$ pornographiques.

Les éléments obligatoires apparaissent quantitativement (ils sont les plus nombreux) et qualitativement (ils apparaissent en premier dans les légendes).

Il apparaît que les pornotypes féminins et ceux référents aux actes organiques sont quasi obligatoires. A contrario, les pornotypes masculins et ceux liés aux orientations sexuelles ou au contexte semblent constituer des circonstances optionnelles.

Qu'elles soient verbales ou nominales, le schéma minimal et profond de ces légendes est quasi immuable dans ses composants et dans son ordre: (1) pornotypes féminins; (2) pornotypes actes organiques; (3) pornotypes masculins; (4) pornotypes liés à la

Les éléments de la scène sont ainsi posés, et l'on pourrait être tenté de voir dans ces légendes une "orientation» du récit, au sens que Labov (1981) donne au terme: un ensemble de propositions libres (mobiles, déplaçables) informant le destinataire sur le lieu, le moment, les personnages, la situation initiale, etc.

D'abord, l'exposé des éléments est trop parcellaire : les personnages sont réduits à des organes ou à des traits physiques, les actions se résument à un ou deux éléments de l'inventaire des pratiques sexuelles ordinaires ou paraphiles... De plus cet exposé parcellaire n'est que très peu informatif en raison de l'extrême récurrence de ses éléments extraits du catalogue des pornotypes.

41 Ensuite, la légende n'est pas une séquence destinée à précéder le récit bien qu'elle se présente comme telle (le récit serait alors déployé dans la production vidéo). Premièrement, parce que la vidéo n'est pas directement accessible (il ne s'agit que d'un extrait). De même parce que le lien entre la légende et l'extrait présenté n'est pas toujours évident. Enfin parce que leur ressemblance diminue leur potentiel informatif.

Il est ainsi difficile de parler de récit.

Michela Marzano en fait le constat écrivant :

Là où l'érotisme est un récit - en images ou en mots - du désir qui pousse un être à la rencontre de l'autre, la pornographie [...] ne vise jamais à raconter une histoire et représente des individus qui ne se reconnaissent pas comme sujets de leur désir.

4 Maingueneau (2007) désigne le phénomène, dans le champ de l'érotisme, comme «pseudo-récit». D’autres auteurs réfutent la dichotomie, telle Williams qui remet en cause la distinction entre (bonne) production érotique et (mauvaise) production pornographique, en soulignant que les frontières se mélangent. Ainsi, de certains films pornographiques qui se présentent comme des productions mixtes où des séquences narratives font le lien entre des scènes pornographiques.

45 Mais dans tous les cas, les vidéos et légendes des sites que nous observons voient la disparition des séquences narratives au profit des scènes exclusivement

6 Il nous semble alors que, contrairement aux apparences, le principal ressort de ces légendes n'est pas d'ordre narratif : il ne s'agit pas de raconter une histoire même sous forme résumée. Leur fonctionnement se rapproche plus du slogan que du récit : il s'agit 
de saisir par la densité sémantique et un réseau de connotations. Pour cela, le principe d'atomisation de la scène est retenu, plutôt que l'union des éléments par l'histoire. On remarquera également la forme proche de celle du slogan, notamment l'emploi de mots pleins, l'élagage syntaxique, la brièveté.

Si l'on préfère, il ne s'agit pas de proposer une histoire cohérente de deux (ou plusieurs) personnages mais de présenter une juxtaposition de traits saillants captatoires et autonomes (organes, actes catégorisés), reconnus par ailleurs car catégorisés sous la forme de pornotypes.

\section{Scripts et fragments}

Les procédés que nous venons de décrire participent à l'interpellation du corps de l'internaute, à une manipulation même, si l'on suit P. Breton (1998), agissant sur les affects (de l'ordre de ce que l'auteur appelle l'« amalgame affectif » et l'« effet fusionnel »). Il faut préciser que nous n'envisageons ici que le dispositif tel qu'il est élaboré par l'émetteur, et que nous ne pouvons, faute de données, prendre en compte l'activité de réception qui ne manquera pas d'en moduler les effets qui n'auront alors rien de massifs et directs.

\subsection{Scripts, scénarios et séquences}

Développé conjointement puis séparément par Gagnon et Simon pendant plus de vingtcinq ans, le concept de "script sexuel » nous invite à concevoir les excitations et conduites sexuelles comme le produit d'une organisation sociale, interpersonnelle et intrapsychique en l'absence duquel «il est improbable qu'il se passe quelque chose de sexuel » $(1973: 17)$.

L'excitation sexuelle ne résulte donc pas - selon ces auteurs - d'une pulsion interne mais de la reconnaissance de schèmes cognitifs mettant en scène des acteurs, des actes, et un contexte structurés. Il est important de souligner que les scripts servent de cadre d'interprétation à la situation potentiellement sexuelle, mais qu'ils constituent aussi des guides comportementaux.

Les scripts apparaissent alors, pour M. Bozon et Giami, comme des séquences narratives :

La perspective des scripts invite à considérer la sexualité en termes de déroulements ou de procédures, dans lesquels les résultats [...] sont obtenus, après que diverses étapes aient été franchies dans un ordre donné, et en respectant les règles d'un environnement socialement structuré (lieu, acteurs, etc.) (1999 : 71). Scénario, séquences, scripts, une organisation narrative semble opérer dans les trois plans où agissent les scripts : intrapsychique, interpersonnel et scénarios culturels.

Les scripts intrapsychiques sont, pour Gagnon, des schèmes cognitifs structurés qui obéissent à une logique narrative qui oriente les projets, les fantasmes. Simon (1974) se démarquera en partie de cette vision narrative qu'il réserve aux scripts interpersonnels et aux scénarios culturels. Pour l'auteur, l'excitation peut être provoquée non pas par l'intention d'avoir des rapports sexuels, mais par des fragments liés à l'histoire personnelle ou issus des médias.

Gagnon trouvera un terrain d'entente :

Les processus mentaux qui organisent les désirs sexuels comportent deux temps:

d'abord, il existe des «fragments » mentaux érotiques et/ou des «émotions » qui 
sont à la source des sensations corporelles érotiques; ensuite, ces éléments sont encodés dans des scripts cognitifs plus organisés qui constituent des instructions pour les interactions concrètes avec d'autres personnes. (1999: 74)

l'est pas. Le scénario peut ne pas être explicitement sexuel et s'enchâsser dans des récits plus larges (ainsi, de nombreuses productions cinématographiques ou littéraires sont vectrices de ces scénarios). Mais le scénario peut aussi présenter des «représentations sexuellement explicites", comme c'est le cas avec les productions de l'industrie pornographique. Ces derniers «ne font pas que spécifier les objets appropriés, les buts et les qualités désirables des relations entre soi et l'autre; ils précisent les moments et les lieux, les séquences de gestes et de propos et surtout ce que l'acteur et (son ou ses) partenaire(s) sont censés ressentir » (Simon et Gagnon 1986 : 105).

Soulignons à ce stade que les scripts nous invitent à concevoir les processus motivant l'excitation et/ou l'activité sexuelle sous l'angle de leurs ressorts sociaux. La dimension narrative apparaît comme prépondérante au plan des scénarios culturels, des relations interpersonnelles et en partie au niveau intrapsychique. La séquentialité apparaît alors comme un trait caractéristique de l'organisation symbolique et sociale de l'excitation et de l'activité sexuelle, pris dans les rets de " scénarios ", " scripts », « récits » qui incitent à penser l'aspect sexuel des relations humaines dans un ajustement comportemental reconnu et partagé.

58 Cette séquentialité, depuis la séduction jusqu'à l'acte, a trouvé son écho dans l'observation des phases de l'acte sexuel.

59 Le modèle de Masters et Johnson (1966), le plus célèbre, se concentre sur les changements physiologiques (vasocongestion et tension musculaire) durant l'acte sexuel pour déterminer des « réponses » sexuelles organisées en phases : excitation, plateau, orgasme, résolution.

60 Ce modèle a connu des développements (par exemple ceux de Kaplan 1979). Pour Gagnon, qui ne remet pas en cause les phases et leur succession, les réponses physiologiques sont motivées par les scripts et ne sont donc pas exclusivement des réactions physiologiques.

61 Reed proposera de compléter les phases en les faisant précéder d'une phase correspondant à l'apparition du désir et en associant aux processus physiologiques des facteurs psychologiques et sociaux : séduction (phases de désir et d'excitation), sensation (phase de plateau), reddition (phase de l'orgasme) et réflexion (phase de résolution).

62 Par nature, ces descriptions articulées à des schémas sont réductrices et caricaturales. Quel que soit le crédit que le lecteur leur accorde (nous n'avons pas compétence pour en juger), elles ont le mérite de souligner une histoire, ou du moins une séquentialité dans l'activité sexuelle (fût-elle onaniste) qui tient autant des réponses physiologiques que des scripts dans lesquels s'inscrit le comportement humain.

63 Ce détour fait, la question se pose de savoir si les sites pornographiques que nous observons sont porteurs de scripts similaires ou semblables à ceux qu'observaient Gagnon et Simon.

Itinéraires, 2014-1 | 2015 


\subsection{Les légendes : du script au fragment?} corps, en une réduction du sujet à sa chair et une réduction de sa chair à l'organe ou l'orifice sur lequel s'appliquent les actes mis en scène comme effecteurs de jouissance. À l'inverse, ses défenseurs expliquent qu'ils ne sont pas exclusifs (Vigouroux rappelle les plans panoramiques ; De Sutter (2006) pense que le style gonzo « adore les plans larges »), ou qu'ils sont du ressort d'une esthétique de l'image que l'on retrouve par ailleurs (dans d'autres films, dans la publicité, dans la photographie).

\section{En guise de conclusion : vers une interpellation émotionnelle}

71 Qu'en est-il dans les sites que nous observons? 
Sur le plan des images, l'utilisation des gros plans dans les vignettes est très fréquente. Nous retrouvons des plans larges mais l'enchevêtrement des personnages vise ici à mettre en avant l'emboîtement des corps et des organes concernés toujours apparents. Si d'autres types de plans apparaissent, variant la monstration de ces organes, ils ne sont toujours qu'accessoires. La réalisation pornographique repose ici sur ces cadrages parcellaires référant comme lieu de la jouissance, au détriment de la narration, du récit.

Sur le plan des mots, on observe (1) une fragmentation du corps et du rapport sexuel à travers les pornotypes que nous avons évoqués et (2) un assemblage de ces "fragments " dans les légendes, sans constituer pour autant un résumé de récit.

Cette opération de fragmentation et de juxtaposition des fragments n'est pas sans rappeler certains phénomènes observés dans le cadre d'échanges "économico-sexuels ». En effet, analysant les cas d'" échanges d'actes sexuels contre de l'argent », Paola Tabet remarque le morcellement de la relation sexuelle en pratiques distinctes au tarif adapté. Dans cette fragmentation de l'échange sexuel, l'auteur voit « un usage global du territoire corporel féminin dans l'indifférence aux exigences de chaque femme » (2003 : 103).

75 On peut également entendre ces mécanismes de fragmentation à la lumière des derniers apports de Simon à la théorie de Gagnon (puis intégrée par ce dernier) que nous évoquions plus haut. Pour les auteurs, certains scripts intrapsychiques échappent à la logique narrative qui est à l'œuvre avec les scripts interpersonnels et dans les scénarios culturels. Rappelons que les "processus mentaux qui organisent les désirs sexuels» (Gagnon 1999: 74) existent dans un premier temps sous la forme de " "fragments" mentaux érotiques et/ou des "émotions" " qui sont lié(e)s à des sensations corporelles érotiques. Ces éléments sont par la suite organisés par les scripts.

Sous forme de lien, dans le cadre des formes contemporaines de la production pornographique que nous observons, nous faisons ici l'hypothèse suivante: les pornotypes (isolés en catégories ou concaténés en légendes) «interpellent » ces traces des sensations fragmentaires en même temps qu'ils offrent ainsi les signes capables de les circonscrire.

7 Si l'on préfère, ils travaillent à l'interface entre des objets sociaux déterminés, partagés, échangeables, et des objets ou motifs sensibles du sujet désirant, en points de projection, « de capiton ", pour reprendre une expression lacanienne (Lacan 1981).

La disparition du récit ou de l'histoire prend ici sens, d'un point de vue stratégique puisque l'interpellation appelle une réponse émotionnelle (réflexe, éruptive, éprouvée) plus qu'émotive (intentionnelle, exprimée, sémiotisée), si l'on reprend la distinction de Plantin ${ }^{3}$ (2003 : 106), ce qui n'exclut pas, bien entendu, qu'elle s'inscrive dans un réseau de signes, scriptés. Le saisissement «à la racine sensible » apparaît donc dans cette stratégie médiatique pornographique, qui propose un espace de projection aux émotions sur des représentations fragmentaires, dans la zone de transaction entre sémiotique et somatique. 


\section{BIBLIOGRAPHIE}

Ariely, Dan et Loewenstein, George, 2006, « The heat of the Moment: The Effect of Sexual Arousal on Sexual Decision Making ", Journal of Behavourial Decision Making, n 19, p. 87-98.

Barthes, Roland, 1964, « Rhétorique de l'image », Communication, vol. 4, n 4, p. 40-51.

Baudry, Patrick, 1997, La Pornographie et ses Images, Paris, Armand Colin.

Bozon, Michel et Giami, Alain, 1999, "Présentation de l'article de John Gagnon », Actes de la recherche en sciences sociales, $\mathrm{n}^{\circ} 128, \mathrm{p} .68-72$.

Breton, Philippe, 1998, La Parole manipulée, Paris, La Découverte.

Caffi, Claudia et Janney, Richard, 1994, « Toward a Pragmatics of Emotive Communication », Journal of Pragmatics, $\mathrm{n}^{\circ}$ 22, p. 325-373.

Gagnon, John H. et Simon, W., 1973, Sexual conduct, Chicago, Aldine.

Gagnon, John H., 1999, « Les usages explicites et implicites de la perspective des scripts dans les recherches sur la sexualité ", Actes de la recherche en sciences sociales, $n^{\circ} 128, p .73-79$. Version originale : 1990, traduit par M. Bozon et C. Cler.

Guillebaud, Jean-Claude, 2011, La Vie vivante : contre les nouveaux pudibonds, Paris, Les Arènes. Ibanez-Bueno, Jacques, 2004, « Ouvertures phénoménologiques sur la télécommunication sexuelle électronique ", Sexe et communication, MEI, n 20, p. 93-106.

Kaplan, Helen Singer, 1979, Disorders of Sexual Desire and Other New Concepts and Techniques in Sex Therapy, New York, Brunner-Mazel.

Lacan, Jacques, 1981, Le séminaire : livre III : Les psychoses (1955-1956), Paris, Seuil, coll. « Le champ freudien ».

—, 1986, Le séminaire, Livre VII, L'éthique de la psychanalyse, Paris, Seuil.

Masters, William et Johnson, Virginia, 1966, Human Sexual Response, Boston, Little, Brown and Company.

Patterson, Zabet, 2004, "Going On-line: Consuming Pornography in the Digital Era », dans L. Williams (dir.), Porn studies, Durham, Duke University Press, p. 104-125.

Paveau, Marie-Anne, 2011, «Signes, sexe and linguistique 2. 69, X, BDSM : des cryptopornèmes ? ", La pensée du discours, [Carnet de recherche], http:// penseedudiscours.hypotheses.org/6100, consulté le 29 décembre 2011.

Perea, François, 2012, «Les sites pornographiques par le menu : pornotypes linguistiques et procédés médiatiques ", Genre, sexualité et société, $\mathrm{n}^{\circ}$ 7, [En ligne], http://gss.revues.org/ index2395.html.

Plantin, Christian, 2003, «Structures verbales de l'émotion parlée et de la parole émue », dans J.M. Colleta et A. Tcherkassof (dir.), Les Émotions. Cognitions, langage et développement, Bruxelles, Mardaga, p. 97-130. 
Simon, William, 1974, « The Social, the Erotic, the Sensual: The Complexities of Sexual Scripts », dans J. K. Cole et R. Dienstbier (dir.), Nebraska Symposium on Motivation, vol. 23, Lincoln, University of Nebraska Press, p. 27-59.

Simon William et Gagnon John H., 1986, « Sexual Scripts: Permanence and Change », Archives of Sexual Behaviour, $\mathrm{n}^{\circ} 15$, p. 97-120.

Sutter, Laurent de, 2006, « Théorie de la fragmentation », Éloge de l'amour, [Blog], mis en ligne le 6 septembre 2006, http://www.elogedelamour-riendespecial.blogspot.com/2006/09/thorie-de-lafragmentation.html, consulté le 20 janvier 2011.

Tabet, Paola, 2004, La Grande arnaque. Sexualité des femmes et échange économico-sexuel, Paris, L'Harmattan, coll. « Bibliothèque du féminisme ».

Tisseron, Serge, 2001, L’Intimité surexposée, Paris, Ramsay.

Vigouroux, Yannick, 2002, « Hybridations et mascarades photographiques : théâtres de l'intime ou esthétique de l'obscène? ", La voix du regard, n 15, p. 197-203.

Williams, Linda, 2004, Porn studies, Durham, Duke university Press.

\section{NOTES}

1. L'histoire enseigne que le terme aurait été utilisé la première fois par le critique A. Thibaudet, en 1923, pour désigner une saisie du monde extérieur par l'écrivain ou le journaliste : il ne s'agit pas de relater son intimité (la scène intérieure du sujet) mais les événements du monde (la scène extérieure). Le psychanalyste J. Lacan s'en saisira pour désigner « ce lieu central, cette extériorité intime, cette extimité, qui est la Chose » (1986: 167), c'est-à-dire un rapport de sublimation inconsciente dont résulte le processus par lequel l'objet va être érigé en signifiant.

2. Pour des raisons juridiques, nous nous abstenons de faire figurer des images de pages d'accueil de sites pornographiques dans cet article.

3. La paire ému/émotionnel de Plantin peut être rapprochée de celle proposée par Caffi et Janney (1994) pour distinguer les cas où l'émotion est contrôlée, affichée, rationnelle et transmises par des signes (communication émotive), de ceux où elle est éprouvée sans volonté ni maîtrise et qui se donne à observer sur des bases indicielles (communication émotionnelle).

\section{RÉSUMÉS}

Le corpus sur lequel repose notre étude est extrait de sites pornographiques particuliers: gratuits et populaires, ils proposent des extraits vidéographiques d'une durée variant de quelques secondes à plusieurs dizaines de minutes. Dans ce contexte, l'image et l'attraction scopique paraissent être les ressorts essentiels et semblent constituer des annihilateurs des autres codes (l'internaute visite le site pour voir). Pourtant, nous remarquons l'importance de ces messages linguistiques (légendes des vignettes, menus des catégories) qui participent de la mise en scène/forme médiatique pornographique. Nous observons d'abord le travail de catégorisation discursive reposant sur un imaginaire et des représentations propres au X (« pornotypes », Perea 2012) des personnages, scènes et actes représentés, en référence aux niches commerciales et au 
dispositif médiatique. Nous poursuivons en interrogeant les légendes des vignettes vidéographiques, qui constituent des motifs narratifs entre scripts (Gagnon et Simon 1986) et pseudo-récits. Nous concluons sur les mécanismes d'interpellation sensible et sémiotique via le matériau linguistique de ces sites.

The corpus of our study is extracted from free and popular private pornsites, which offer short videographic extracts. In this context, the image and the visual attraction appear to be essential and seems to be annihilators of other codes (the user visits the site to see). However, we note the importance of the linguistic messages (captions, menu "categories") involved in the pornographic media form. We first observe the discursive work of categorization based on an imaginary and representations produces by the X ("pornotypes", Perea 2012): characters, scenes and acts represented, with reference to niche markets and media device. We continue asking captions, which are narrative motifs between scripts (Gagnon and Simon 1986) and pseudonarratives. We conclude on the mechanisms of sensitive and semiotic inquiry via the linguistic material from these sites.

\section{INDEX}

Keywords : pornography, pornotypes, captions, discourse, image

Mots-clés : pornographie, pornotypes, legendes, discours, image

\section{AUTEUR}

\section{FRANÇOIS PEREA}

Université Paul-Valéry - Montpellier 3, CNRS, Praxiling (UMR 5267) 